\title{
Preparation and characteristics of lipid nanoemulsion formulations loaded with doxorubicin
}

This article was published in the following Dove Press journal:

International Journal of Nanomedicine

17 August 2013

Number of times this article has been viewed

\author{
Sai-Ping Jiang ${ }^{1,2, *}$ \\ Sai-Nan $\mathrm{He}^{3, *}$ \\ Yun-Long $\mathrm{Li}^{2,3}$ \\ Da-Lin Feng ${ }^{2}$ \\ Xiao-Yang Lu' \\ Yong-Zhong $\mathrm{Du}^{2}$ \\ $\mathrm{He}$-Yong $\mathrm{Yu}^{3}$ \\ Fu-Qiang $\mathrm{Hu}^{2}$ \\ Hong Yuan ${ }^{2}$ \\ 'Department of Pharmacy, \\ The First Affiliated Hospital, College \\ of Medicine, Zhejiang University, \\ Hangzhou, ${ }^{2}$ College of Pharmaceutical \\ Sciences, Zhejiang University, \\ Hangzhou, ${ }^{3}$ Women's Hospital, School \\ of Medicine, Zhejiang University, \\ Hangzhou, People's Republic of China \\ *These authors contributed equally \\ to this work
}

Purpose: Safe and effective lipid nanoemulsion (LNE) formulations for the antitumor delivery of doxorubicin is designed.

Methods: LNEs composed of medium-chain triglyceride, soybean oil, lecithin, and doxorubicin are prepared by a solvent-diffusion method in an aqueous system. The effects of lipid material composition and polyethylene glycol (PEG)ylation on the size, drug encapsulation efficiency, and stability of LNEs are investigated. Based on in-vitro cytotoxicity and cellular uptake tests of A549 (human lung carcinoma) cells, in-vivo biodistribution, antitumor activity, and cardiac toxicity are further examined using nude mouse bearing A549 tumor.

Results: The LNE size decreases from $126.4 \pm 8.7 \mathrm{~nm}$ to $44.5 \pm 9.3 \mathrm{~nm}$ with increased weight ratio of medium-chain triglyceride to soybean oil from 1:4 to $3: 2$, whereas the encapsulation efficiency of doxorubicin is slightly reduced from $79.2 \% \pm 2.1 \%$ to $71.2 \% \pm 2.9 \%$. The PEGylation of LNE by 1,2-distearoyl-sn-glycero-3-phosphoethanolamine-N-[carboxy(PEG)2000] (DSPE-PEG 2000) does not significantly change the size and drug encapsulation efficiency. Three-month storage at room temperature and lyophilization process does not affect the drug encapsulation efficiency, whereas the size slightly increases to almost $100 \mathrm{~nm}$. The in-vitro drug-release profiles of LNEs suggest that the present formulation can prolong drug release for 48 hours. LNEs can be internalized into tumor cells in vitro and efficiently accumulate in tumor tissues in vivo by passive targeting. Analysis results of in-vitro and in-vivo antitumor activities reveal that doxorubicin-loaded LNE exerts a therapeutic effect similar to that of the commercial Adriamycin. Moreover, the toxicity of doxorubicin, particularly its cardiac toxicity, is reduced.

Conclusion: The present LNE formulation of doxorubicin can effectively suppress tumor growth and improve the safety of Adriamycin.

Keywords: PEGylation, stability, antitumor activity

\section{Introduction}

Drug delivery systems (DDSs) have been extensively studied in recent years. Compared with traditional drug preparations, DDSs can directly deliver the drug to its designated location, improve therapeutic efficacy, and subsequently reduce side effects. Lipid nanoemulsions (LNEs) as a new kind of DDS have been prepared from soybean oil, triglyceride, and egg yolk lecithin. These LNEs were initially used to supply energy to patients. ${ }^{1}$ LNEs are also promising vehicles for drug administration because of their features of sustained release, targeted delivery, as well as reduced irritation and drug toxicity. ${ }^{2-4}$ LNEs with an oil-like lipid matrix are commonly prepared by incorporating drugs into the interior oil phase or into the oil-water interface. ${ }^{5}$ Therefore, unstable drugs with poor aqueous
Correspondence: Hong Yuan College of Pharmaceutical Sciences, Zhejiang University, 866 Yuhangtang Road, Hangzhou 310058,

People's Republic of China

Tel +8657188208439

Fax +8657188208439

Email yuanhong70@zju.edu.cn 
solubility can be loaded into the interior oil phase of an LNE, thereby minimizing hydrolysis of these drugs. ${ }^{6}$

A specific type of lipid nanoparticles known as longcirculating lipid nanoparticles has been developed. These nanoparticles can circulate in the blood for long periods without being trapped by the mononuclear phagocytic system. ${ }^{7,8}$ These nanoparticles, which contain a certain amount of hydrophilic chains on the surface, are usually prepared using polyethylene glycol (PEG)-derivatized phospholipids and are thus lowly susceptible to uptake by the reticuloendothelial system. ${ }^{9,10}$ The ability of PEG derivatives to prolong the circulation time of nanoparticles depends on the PEG chain length (PEG $2000>$ PEG $5000>$ PEG 1000) and on the PEG density on nanoparticle surface. ${ }^{11,12}$ Nanoparticle PEGylation can alter the pharmacokinetic properties of a drug, reduce the drug volume distribution, prolong the exposure of antitumor drugs to tumor cells, and increase the effect of enhanced permeability and retention (EPR). ${ }^{13,14}$ The characteristics of a loaded drug can also markedly influence the capacity of PEG-coated nanoparticles in increasing the plasma residence of a drug. ${ }^{15}$

In the present study, an LNE formulation was designed using doxorubicin (DOX) as a model antitumor drug. The LNE, which was composed of medium-chain triglyceride (MCT), soybean oil, lecithin, and DOX, was prepared by a solvent diffusion method in an aqueous system. 1,2-distearoylsn-glycero-3-phosphoethanolamine-N-[carboxy(polyethylene Glycol)2000] (DSPE-PEG 2000) was incorporated into LNE to obtain PEGylated LNE. The physicochemical characteristics of the LNE, such as size, zeta potential, drug encapsulation efficiency, drug-release profiles, and stabilities of LNE and PEGylated LNE were evaluated. Thereafter, the in-vitro cellular uptake, cytotoxicity, and in-vivo biodistribution and antitumor activities of LNE and PEGylated LNE were also evaluated.

\section{Materials and methods Materials}

DOX hydrochlorate $(\mathrm{DOX} \cdot \mathrm{HCl})$ was provided by Hisun Pharm Co, Ltd (Zhejiang, People's Republic of China). Fluorescein isothiocyanate (FITC) and 3-(4,5-dimethylthiazol-2yl)-2,5-diphenyltetrazolium bromide (MTT) were purchased from Sigma-Aldrich (St Louis, MO, USA). Octadecylamine (ODA) was purchased from Fluka Co, Ltd (Fluka/SigmaAldrich, St Louis, MO, USA). MCT, lecithin $\left(\mathrm{E}_{80}\right)$, and DSPE-PEG 2000 were purchased from Lipoid Co, Ltd (Ludwigshafen, Germany). Soybean oil was acquired from KeXing Laboratory Equipment Co, Ltd (Shanghai, People's Republic of China). Cremophor EL was purchased from Aladdin Chemistry Co, Ltd (Shanghai, People's Republic of China). DiR (1,1'-dioctadecyl-3,3,3',3'-tetramethyl indotricarbocyanine iodide) was purchased from Invitrogen/Life Technologies (Carlsbad, CA, USA). Poloxamer 188 was purchased from Jiqi Pharmaceutical Industry of Shen Yang Pharmaceutical University Co, Ltd (Shen Yang, People's Republic of China). Mannitol was provided by Dongsheng Chemical Industry Reagent Co, Ltd (Zhejiang Wenzhou, People's Republic of China). Anhydrous dimethyl sulfoxide (DMSO), diethyl ether, and ethanol were obtained from Haishuo Biochemistry Co, Ltd (Wuxi, People's Republic of China). Other chemicals were analytical reagent grade.

\section{Synthesis of ODA-FITC conjugate}

ODA was dissolved in ethanol, and the same molar amount of FITC was added under stirring. After 24 hours of reaction in darkness, ODA-FITC was mixed with a tenfold volume of distilled water and passed through a $0.45 \mu \mathrm{m}$ Millipore (Merck Millipore, Billerica, MA, USA) filter. Distilled water was used to wash the product five times. The obtained conjugate (ODAFITC) on the membrane was dried at room temperature under sealed conditions and stored in the dark for further use.

\section{Preparation of DOX-loaded LNEs}

DOX was obtained by the reaction of $\mathrm{DOX} \cdot \mathrm{HCl}$ with twice the molar amount of triethylamine in DMSO. In a typical procedure, $\mathrm{DOX} \cdot \mathrm{HCl}$ was dissolved in $10 \mathrm{~mL}$ of deionized water, and then triethylamine was added. The reaction was conducted in a dark place for 2 hours. The mixed solution was subjected to overnight dialysis (molecular weight cutoff [MWCO], 3500 Da; Spectrum Laboratories, Laguna Hills, CA, USA) against deionized water. Finally, the solution was centrifuged at $4000 \mathrm{rpm}$ for 10 minutes. The precipitate was then washed and lyophilized to obtain DOX.

DOX-loaded LNEs (DOX/LNE) and PEG-modified LNEs (DOX/PEG/LNE) were prepared by a solvent-diffusion method in an aqueous system as reported in our previous study. ${ }^{16,17}$ In a typical procedure, MCT, soybean oil, lecithin, DSPE-PEG, and DOX were dissolved in a mixed solvent of DMSO and ethanol $(1 / 1, \mathrm{v} / \mathrm{v})$. The resultant organic solution was heated to $70^{\circ} \mathrm{C}$ and then quickly dispersed in distilled water under mechanical agitation at $400 \mathrm{rpm}$ for 5 minutes in a $70^{\circ} \mathrm{C}$ water bath. After adjusting the $\mathrm{pH}$ of the dispersion to 1.2 by adding hydrochloric acid, the precipitate of LNE was centrifuged ( $3 \mathrm{~K} 30$, Sigma, Germany) at 24,000 rpm for 20 minutes under $4^{\circ} \mathrm{C}$. The LNE precipitate was redispersed in distilled water after washing twice with deionized water.

To lyophilize LNE, an aqueous solution for dissolving the cryoprotectant mannitol and poloxamer 188 was added to the 
resultant redispersion, and the mixture was placed in a deep freezer (Sanyo Ultra-Low Temperature Freezer MDF-192, Japan) at $-75^{\circ} \mathrm{C}$ for 5 hours and then rapidly moved to a freeze drier (Freezone 2.5 L; LABCONCO, Kansas City, MO, USA). The obtained powder was collected, weighed, and stored at room temperature. The residual organic solvent during LNE lyophilization was determined by gas chromatography (Agilent 6890N).

\section{Characterization of LNEs}

The size and zeta potential of LNE were measured with Zetasizer (3,000 HS; Malvern Instruments, Malvern, UK). The sample was prepared by diluting the LNE resuspension to $100 \mu \mathrm{g} / \mathrm{mL}$ lipid concentration. Morphological examinations were performed using a transmission electron microscopy (TEM) system (JEM-1230; JEOL, Tokyo, Japan). The samples were diluted to $0.2 \mathrm{mg} \cdot \mathrm{mL}^{-1}$, dropped onto a carbon-coated copper grid, and stained with $2 \%(\mathrm{w} / \mathrm{v})$ phosphotungstic acid for viewing under TEM.

The DOX content was determined with a fluorescence spectrophotometer (F-2500; Hitachi Ltd, Tokyo, Japan). The drug encapsulation efficiency (EE\%) and drug-load content (DL\%) of the LNE were then indirectly determined. The prepared LNE dispersion was passed through an ultrafiltration tube (MWCO, 10,000 Da; Merck Millipore, Billerica, MA, USA) at 15,000 rpm for 30 minutes. The amount of DOX in the filtrate was measured with a fluorescence spectrophotometer. The excitation wavelength, emission wavelength, and slit openings were set at 505, 565, and $5 \mathrm{~nm}$, respectively. The EE\% and DL\% were calculated using Equations 1 and 2, respectively.

$$
\begin{aligned}
& \text { EE } \% \\
& =\frac{(\text { Total weight of DOX }- \text { Weight of DOX in supernatants })}{\text { Total weight of DOX }} \\
& \quad \times 100 \% \quad \text { DL } \% \quad \text { (Weight of lipid + Total weight of DOX } \\
& =\frac{(\text { Total weight of DOX }- \text { Weight of DOX in supernatants })}{- \text { Weight of DOX in supernatants })} \\
& \quad \times 100 \% \quad
\end{aligned}
$$

\section{Drug-release test from LNE}

In-vitro cumulative drug release of DOX from LNE was investigated using phosphate-buffered saline (PBS) ( $\mathrm{pH} 7.4$ ) as a release medium. DOX, DOX/LNE, and DOX/PEG/ LNE containing $50 \mu \mathrm{g}$ of DOX were suspended in $1 \mathrm{~mL}$ of deionized water and poured into a dialysis membrane bag (MWCO, 7000 Da; Spectrum Laboratories) in plastic tubes. Then, $15 \mathrm{~mL}$ of PBS (the saturated solubility of DOX in PBS was determined to be about $20 \mu \mathrm{g} / \mathrm{mL}$, which reached the sink condition) was added. The plastic tubes were then placed in an incubator shaker and shaken horizontally at $60 \mathrm{rpm}$ at $37^{\circ} \mathrm{C}$. The solution in the tube was periodically withdrawn and completely replaced with an equal volume of fresh PBS at predefined time intervals. The concentration of DOX was determined with a fluorescence spectrophotometer (F-2500; Hitachi Ltd).

\section{Stability test of LNE}

The storage stabilities of DOX/LNE and DOX/PEG/LNE were also determined. A certain volume of LNE dispersion solution was stored at room temperature under sealed conditions; the mean size, zeta potential, and $\mathrm{EE} \%$ were investigated once a month for 3 months. For comparison, lyophilized samples were also stored under the same conditions. Before measurements, the powders of the samples were redispersed in distilled water with $1 \mathrm{mg} \cdot \mathrm{mL}^{-1}$ concentration by vortexing (XW-80A; Instruments factory of Shanghai Medical University) for 20 seconds.

\section{Cell culture}

A549 (human lung carcinoma) cells were obtained from the Cell Resource Center of China Science Academy. Cells were maintained in RPMI (Roswell Park Memorial Institute) 1640 $\left(\right.$ Gibco $^{\circledR}$; Life Technologies) supplemented with 10\% (v/v) newborn calf serum (Sijiqing Biologic, Hangzhou, People's Republic of China) and penicillin/streptomycin (100 $\mathrm{U} \mathrm{mL}^{-1}$ each) at $37^{\circ} \mathrm{C}$ in a humidified incubator with an atmosphere of $5 \% \mathrm{CO}_{2}$. Cells were regularly subcultured using trypsin/ EDTA.

\section{In-vitro cytotoxicity assay}

The in-vitro cytotoxicity of DOX (the positive control), DOX/LNE, and DOX/PEG/LNE against A549 cells were evaluated by MTT assay. Cells were seeded at a density of 10,000 cells per well in a 96-well plate (Nalge Nunc International, Naperville, IL, USA) and incubated for 24 hours. After pre-incubation, cells were exposed to DOX, $\mathrm{DOX} / \mathrm{LNE}$, and DOX/PEG/LNE solution with serial concentrations for another 48 hours. At predetermined time points, $20 \mu \mathrm{L}$ of MTT solution $\left(5 \mathrm{mg} \cdot \mathrm{mL}^{-1}\right)$ was added followed by incubation for 4 hours. Then, the metabolized product 
MTT formazan was dissolved by adding $200 \mu \mathrm{L}$ of DMSO to each well. Finally, the plate was shaken for 20 minutes, and the absorbance of the formazan product was measured at $570 \mathrm{~nm}$ in a microplate reader (Bio-Rad, Model 680; Bio-Rad Laboratories, Hercules, CA, USA).

\section{Cellular uptake tests}

For the cellular uptake experiment, $5 \%(\mathrm{w} / \mathrm{w})$ of lipid in the preparation of LNE was replaced with ODA-FITC to prepare FITC-labeled DOX/LNE or DOX/PEG/LNE. A549 cells were seeded at a density of $1.0 \times 10^{5}$ cells $/ \mathrm{mL}$ onto a 24-well culture plate and incubated for 24 hours. The medium containing FITC-labeled DOX/LNE or DOX/ PEG/LNE was added to the well before fluorescence images observation at 1,6 , and 12 hours respectively, and then incubated for 1, 6, and 12 hours. After the incubation, the cells were washed twice with PBS ( $\mathrm{pH} 7.4)$, and the cellular uptake of FITC-labeled LNE was viewed by fluorescence microscopy (Olympus America, Melville, NY, USA).

\section{Establishment of a tumor-bearing animal model}

All animal procedures were approved by the Zhejiang University Institutional Animal Care and Use Committee. BALB/C+nu/F1 nude mice (6-8 weeks old) were transplanted with A549 cells by subcutaneous injection of $5.0 \times 10^{5}$ cells, and then raised for a period of time until needed for further use.

\section{In-vivo imaging}

For in-vivo fluorescence imaging, DiR was used as a molecular probe. ${ }^{18}$ About $100 \mu \mathrm{g}$ of DiR was loaded into $50 \mathrm{mg}$ of lipid material to obtain the DiR/lipid materials and DiR/PEG/LNE. The final concentration of LNE was $6 \mathrm{mg} \cdot \mathrm{mL}^{-1}$. A549 tumor-bearing nude mice were injected intravenously through the tail veil at a dose of $0.2 \mathrm{~mL} \mathrm{DiR/}$ LNE. At multiple time points, the mice were anesthetized, and images were collected using the Maestro In Vivo Imaging System (CRI Inc, Woburn, MA, USA). The tunable filter was automatically stepped in $10 \mathrm{~nm}$ increments from 580 to $700 \mathrm{~nm}$, whereas the camera captured images at each wavelength with constant exposure. Overall, the acquisition time was about 0.5 seconds. For ex-vivo imaging, after tumors and hearts were harvested, tissues were immediately subjected to fluorescence imaging using the Spectral Imaging System. Moreover, 1\% (v/v) of the injected amount of DiR/LNE was used as standard point to semi-quantify.
The semi-quantitative content in each organ or tumor was calculated using Equation 3.

$$
\begin{aligned}
& \text { DiR/LNE in tissue }(\%) \\
& =\frac{(\text { The intensity of different tissues })}{(\text { The intensity of the standard point }) \times 100} \times 100 \%
\end{aligned}
$$

\section{In-vivo antitumor activity of DOX-loaded LNEs}

Different formulations were injected through the tail vein when the tumor volume reached approximately $100 \mathrm{~mm}^{3}$. Mice were randomly divided into four groups, with six mice per group. The first group, which served as the negative control, was injected with $0.2 \mathrm{~mL}$ of $0.9 \%$ saline for 7 days consecutively. The second group, which served as the positive control, was injected with $2 \mathrm{mg} \cdot \mathrm{kg}^{-1}$ body weight of Adriamycin (commercial DOX hydrochloride injection) for 7 days consecutively. The third and fourth groups were injected with DOX-loaded LNE $\left(2 \mathrm{mg} \cdot \mathrm{kg}^{-1}\right.$ equivalent DOX and $4 \mathrm{mg} \cdot \mathrm{kg}^{-1}$ equivalent DOX, respectively) for 7 days consecutively.

The size of the tumor and bodyweight of each mouse were monitored every 3 days thereafter. After 27 days, mice were sacrificed and tumor weight was measured. Tumor volume and inhibition of tumor growth (\%) were calculated using Equations 4 and 5.

Tumor volume

(The smallest diameter $) \times($ The smallest diameter $)$

$$
=\frac{\times(\text { The largest diameter })}{2}
$$

Inhibition of tumor growth (\%)

$$
\begin{aligned}
= & 1-\frac{(\text { The average tumor weight of treated group) }}{(\text { The average tumor weight of negative control group) }} \\
& \times 100 \%
\end{aligned}
$$

\section{Evaluation of the cardiac toxicity of DOX-loaded LNEs}

The cardiac toxicity of DOX-loaded LNEs was studied by comparing with free DOX. BALB/c mice were randomized into four groups $(n=5)$. One group was untreated, and the other three were injected intravenously with a single dose of $0.9 \%$ saline, free DOX (10 mg/kg), and DOX/LNE 
Table I Preparation recipes and characteristics of LNE with different weight ratio of soybean oil to MCT

\begin{tabular}{|c|c|c|c|c|c|c|c|}
\hline & \multicolumn{4}{|c|}{ Materials (mg) } & \multicolumn{3}{|c|}{ Physicochemical properties } \\
\hline & Soybean oil & MCT & Lecithin & $d_{n}(n m)$ & Zeta potential $(\mathrm{mV})$ & EE (\%) & DL (\%) \\
\hline A & 80 & 20 & 12 & $126.4 \pm 8.7^{*}$ & $-22.4 \pm 4.6$ & $79.2 \pm 2.1$ & $4.0 \pm 0.1$ \\
\hline B & 60 & 40 & 12 & $97.2 \pm 7.6^{*}$ & $-27.4 \pm 5.2$ & $78.5 \pm 3.3$ & $4.0 \pm 0.2$ \\
\hline C & 50 & 50 & 12 & $56.2 \pm 7.6^{*}$ & $-26.3 \pm 3.5$ & $75.2 \pm 1.4$ & $3.8 \pm 0.1$ \\
\hline D & 40 & 60 & 12 & $44.5 \pm 9.3^{*}$ & $-25.6 \pm 7.3$ & $71.2 \pm 2.9$ & $3.6 \pm 0.1$ \\
\hline$E$ & 20 & 80 & 12 & $64.8 \pm 8.7^{*}$ & $-27.8 \pm 6.1$ & $66.2 \pm 3.8$ & $3.3 \pm 0.2$ \\
\hline
\end{tabular}

Notes: Data are expressed as the mean \pm standard deviation $(n=3)$; ${ }^{*}$ indicates significant difference $(P<0.05)$.

Abbreviations: LNE, lipid nanoemulsion; MCT, medium-chain triglyceride; $d_{n}$, hydrodynamic diameter of LNE; EE (\%), drug encapsulation efficiency; DL (\%), drug loading content.

$(10 \mathrm{mg} / \mathrm{kg}$ ) on day 0 . On day 27 , the mice were euthanized and then their hearts were immediately harvested and fixed in $4 \%$ formalin. Thereafter, the hearts were incrementally dehydrated by increasing concentrations of alcohol and then stained with hematoxylin and eosin, followed by microscopic examination of cardiomyopathy.

\section{Statistical analysis}

Data were expressed as the mean of three separate experiments. The significances of differences were determined using Student's $t$-test two-tailed for each paired experiment. $P<0.05$ was considered statistically significant in all cases.

\section{Results and discussion Preparation and characteristics of LNE} Ethanol and DMSO were used to prepare LNE. DMSO was used because of the poor solubility of DOX in ethanol. Moreover, mixed organic solvent of DMSO and ethanol was used to completely dissolve DOX and lipid materials. When the mixed solution dissolving with DOX and lipid materials was added to the water phase, the mixed solvent quickly diffused into the aqueous phase, and DOX and lipid materials consequently separated from the aqueous phase and formed DOX-loaded LNE.

Table 1 lists the preparation steps and characteristics of LNE with different weight ratios of soybean oil to MCT.
The nanoemulsion size significantly decreased from $126.4 \pm 8.7 \mathrm{~nm}$ to $44.5 \pm 9.3 \mathrm{~nm}$ after the weight ratio of MCT to soybean oil increased from 1:4 to $3: 2$. However, the nanoemulsion size increased again when the weight ratio of MCT to soybean oil was further increased to $4: 1$. Furthermore, the drug encapsulation efficiency decreased from $79.2 \% \pm 2.1 \%$ to $71.2 \% \pm 2.9 \%$ with increased weight ratio of MCT to soybean oil from $1: 4$ to $3: 2$ and then decreased to $66.2 \% \pm 3.8 \%$ when the weight ratio of MCT to soybean oil was increased to $4: 1$. The reduced drug encapsulation efficiency can be attributed to the decreased amount of soybean oil, which had better solubility for DOX than MCT. Considering the characteristics of particle size and drug encapsulation efficiency, formula $\mathrm{C}$ was chosen for further research because of its relatively smaller particle size and higher drug encapsulation efficiency. The drug-loading content of formula $\mathrm{C}$ (Table 1) was $3.8 \% \pm 0.1 \%$.

To obtain PEG-modified LNE, 50 weight (wt)\% of lecithin in formula $\mathrm{C}$ was replaced by DSPE-PEG. The size of LNE increased from $56.2 \pm 7.6 \mathrm{~nm}$ to $64.8 \pm 8.7 \mathrm{~nm}$ after PEGylation, whereas the drug encapsulation efficiency and zeta potential had no significant change (Table 2). The increased size can be attributed to the hydrophilic PEG chain on the emulsion surface in the water phase. Figure 1 shows the size distribution as determined by dynamic light scattering and TEM images of DOX/LNE and DOX/PEG/LNE. Spherical morphology can be observed from Figure 1C and D.

Table 2 Physical stability of DOX/LNE and DOX/PEG/LNE and their freeze-dried samples

\begin{tabular}{|c|c|c|c|c|c|c|c|c|}
\hline & \multicolumn{2}{|l|}{$d_{n}(n m)$} & \multicolumn{2}{|c|}{ Zeta potential $(\mathrm{mV})$} & \multicolumn{2}{|l|}{ EE (\%) } & \multicolumn{2}{|l|}{ DL (\%) } \\
\hline & 0 day & 3 months & 0 day & 3 months & 0 day & 3 months & 0 day & 3 months \\
\hline DOX/LNE & $56.2 \pm 7.6$ & $83.5 \pm 12.2$ & $-26.3 \pm 3.5$ & $-22.0 \pm 4.2$ & $75.2 \pm 1.4$ & $73.2 \pm 2.5$ & $3.8 \pm 0.1$ & $3.7 \pm 0.1$ \\
\hline DOX/PEG/LNE & $64.8 \pm 8.7$ & $117 \pm 18.3$ & $-21.2 \pm 4.4$ & $-16.8 \pm 3.4$ & $72.3 \pm 3.2$ & $71.3 \pm 2.5$ & $3.6 \pm 0.2$ & $3.6 \pm 0.1$ \\
\hline L-DOX/LNE & $76.3 \pm 12.3$ & $94.3 \pm 21.1$ & $-28.4 \pm 4.2$ & $-28.4 \pm 5.1$ & $74.8 \pm 2.3$ & $74.3 \pm 1.3$ & $3.7 \pm 0.1$ & $3.7 \pm 0.1$ \\
\hline L-DOX/PEG/LNE & $87.5 \pm 8.7$ & $116 \pm 12.1$ & $-23.8 \pm 6.7$ & $-28.9 \pm 3.2$ & $71.8 \pm 2.4$ & $70.2 \pm 2.6$ & $3.6 \pm 0.1$ & $3.5 \pm 0.1$ \\
\hline
\end{tabular}

Notes: Data are expressed as the mean \pm standard deviation $(n=3)$; L-DOX/LNE and L-DOX/PEG/LNE were the lyophilized samples of DOX/LNE and DOX/PEG/LNE. Abbreviations: DOX, doxorubicin; LNE, lipid nanoemulsion; PEG, polyethylene glycol; $d_{n}$, hydrodynamic diameter; EE (\%), drug-encapsulation efficiency; DL (\%), drug-loading content. 


\section{A Size distribution(s)}
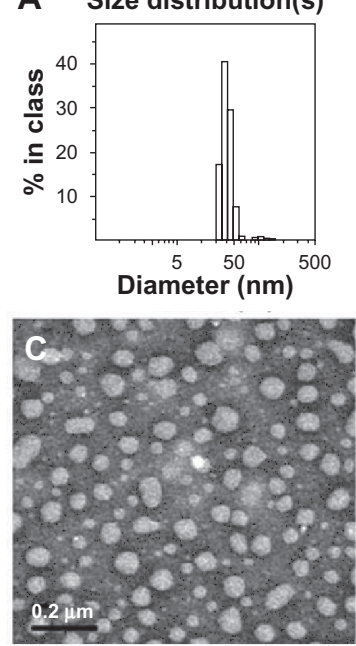

Figure I Size distribution and TEM images of DOX/LNE and DOX/PEG/LNE. (A) Size distribution of DOX/LNE; (B) Size distribution of DOX/PEG/LNE; (C) TEM image of DOX/LNE; (D) TEM image of DOX/PEG/LNE.

Abbreviations: TEM, transmission electron microscopy; DOX, doxorubicin; LNE, lipid nanoemulsion; PEG, polyethylene glycol.

Table 2 shows the physicochemical properties of lyophilized samples of DOX/LNE and DOX/PEG/LNE before and after storing samples at room temperature for 3 months. The lyophilization process using mannitol and poloxamer 188 as the cryoprotectant did not significantly influence the drug encapsulation efficiency of DOX-loaded LNE and PEGylated LNE. However, the size of DOX/PEG/LNE and DOX/LNE increased from $64.8 \pm 8.7 \mathrm{~nm}$ to $87.5 \pm 8.7 \mathrm{~nm}$ and $56.2 \pm 7.6 \mathrm{~nm}$ to $76.3 \pm 12.3 \mathrm{~nm}$, respectively after lyophilization. The increased size may be attributed to the effect of the lyophilization process that led to the coagulation of small LNEs; however, all sizes of LNEs were $<90 \mathrm{~nm}$. The stability test of LNE dispersion solution also indicated that drug encapsulation efficiency had no apparent changes at room temperature for 3 months; however, the particle size especially of DOX/PEG/LNE increased from $64.8 \pm 8.7 \mathrm{~nm}$ to $117 \pm 18.3 \mathrm{~nm}$ after 3 months. This increase can be associated with the decreased zeta potential (from $-21.2 \pm 4.4 \mathrm{mV}$ to $-16.8 \pm 3.4 \mathrm{mV}$ ) during the stability test and caused the aggregation of LNEs. The residual organic solvent in the lyophilization of LNE was determined by gas chromatography (Agilent $6890 \mathrm{~N}$ ). No ethanol was determined, and the residual DMSO concentration was $12.3 \pm 1.2 \mathrm{ppm}$, which was much lower than the $0.5 \%$ restricted value of DMSO in injection preparation.

In-vitro drug-release profiles of DOX/LNE and DOX/ $\mathrm{PEG} / \mathrm{LNE}$ are shown in Figure 2. The saturation solubility of DOX in PBS (pH 7.4) was determined to be about $20 \mu \mathrm{g} / \mathrm{mL}$, the applied concentration of DOX was $2.5 \mu \mathrm{g} / \mathrm{mL}$; thus, a good sink condition was maintained during drug-release tests. Figure 2 shows that both samples released about $80 \%$ of the drug after 48 hours, and no significant difference was found between the release profiles of DOX/LNE and DOX/ PEG/LNE. Drugs released from LNE were probably related to the mechanism of diffusion.

\section{In-vitro cellular uptake}

Figure 3 shows the fluorescence images of A549 cells incubated with FITC/LNE or FITC/PEG/LNE for 2, 6, and

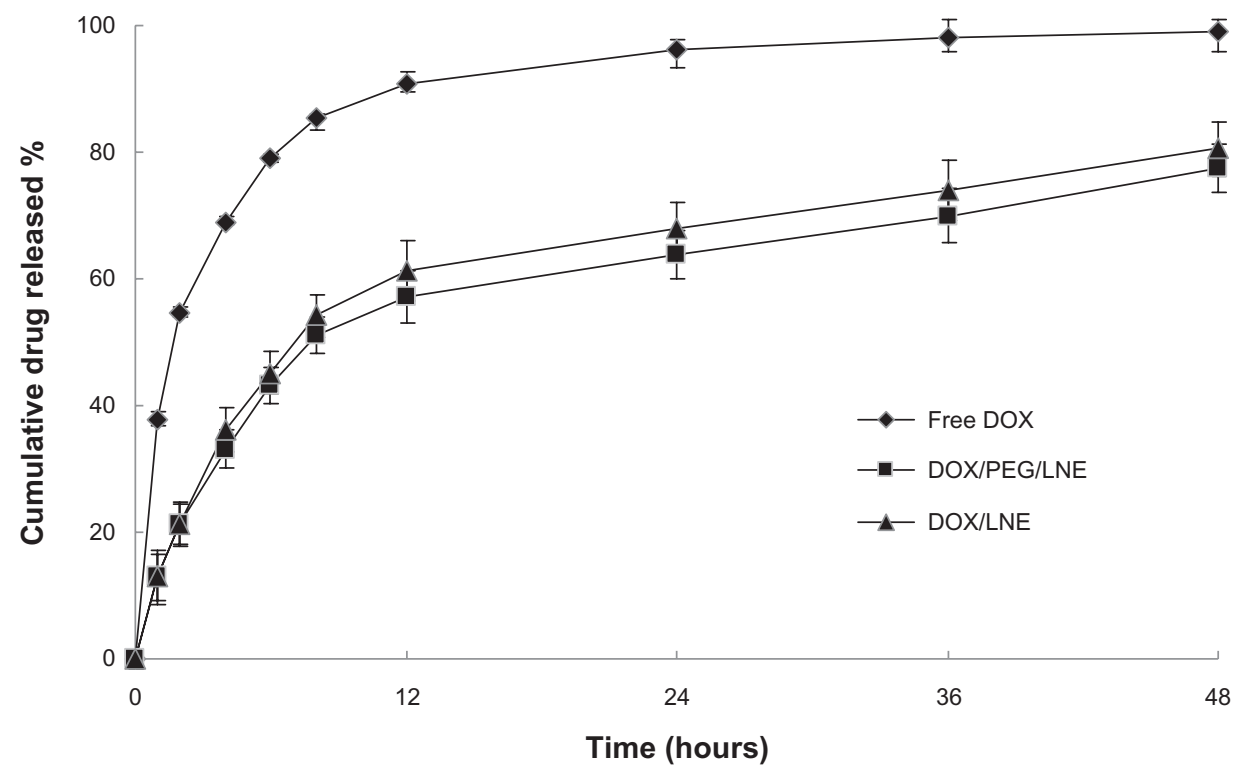

Figure 2 In-vitro drug-release profiles of free DOX, DOX/LNE, and DOX/PEG/LNE in pH 7.4 phosphate-buffered saline (PBS) at $37^{\circ} \mathrm{C}$. Abbreviations: DOX, doxorubicin; LNE, lipid nanoemulsion; PEG, polyethylene glycol. 


\section{1 hour}
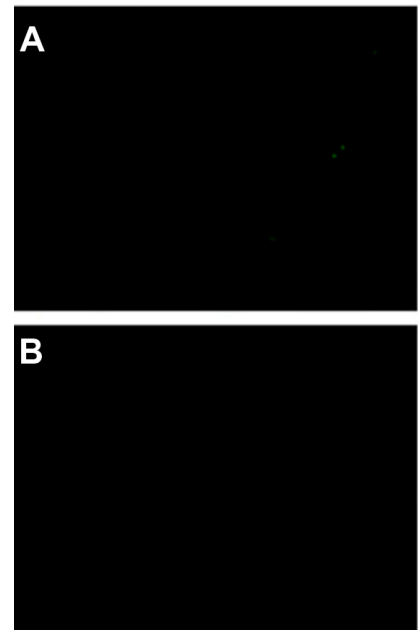

6 hours
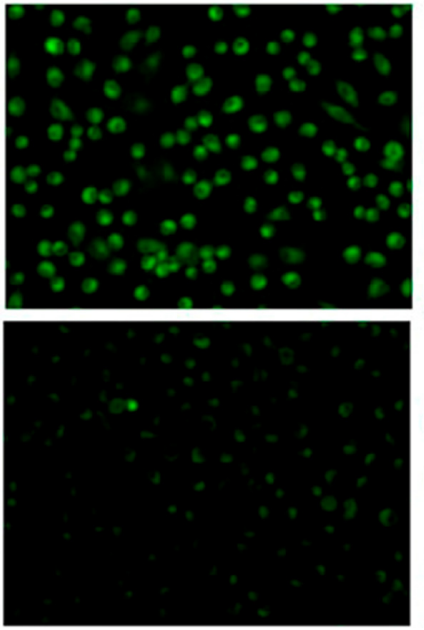

12 hours
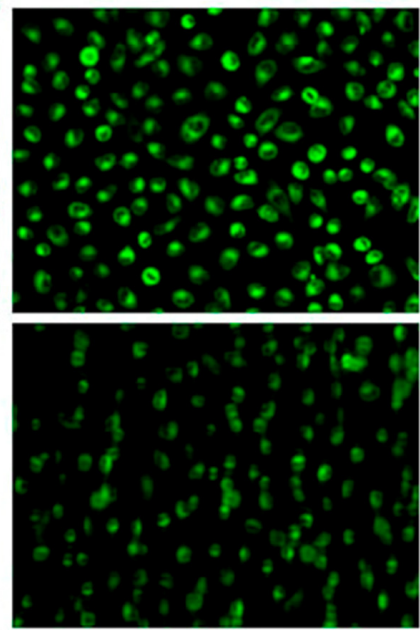

Figure 3 Fluorescence images after the A549 cells were exposed to FITC-labeled LNE for I, 6, and I2 hours, respectively. Notes: The lipid concentration of LNE was $50 \mu \mathrm{g} \cdot \mathrm{mL}^{-1}$. Co-cultured with PEG-modified LNE (A); and nonmodified LNE (B). Abbreviations: FITC, fluorescein isothiocyanate; LNE, lipid nanoemulsion; PEG, polyethylene glycol.

12 hours, respectively. The fluorescence intensity increased with increased incubation time, which indicated that the cellular uptake of LNE was time dependent.

Over the past decades, several efforts have been devoted to the surface modification of various drug carriers with PEG to improve the internalization efficiency. PEG modification can enhance the cell membrane crossing ability of nanoparticles. In a previously reported mechanism, PEG can be dissolved in both polar and nonpolar solvents and has a high solubility in cell membranes. ${ }^{19}$ Other researchers have also reported a hydration shell that exists around the cell membrane, and this shell prevents particles whose surface is excessively lipophilic, from approaching the cell membrane. This excessive hydrophilicity can prevent the internalization of nanoparticles into cells, and PEG modification can balance the hydrophilicity and lipophilicity of the particle surface and enhance uptake. ${ }^{8,20-22}$ Figure 3 shows that the cellular uptakes of LNE were highly enhanced by the incorporation of DSPE-PEG into LNE.

\section{In-vitro cytotoxicity}

The variations in cell viability against the drug concentration of free DOX, DOX/LNE, and DOX/PEG/LNE solution are presented in Figure 4. The $\mathrm{IC}_{50}$ values of DOX/LNE and DOX/PEG/LNE against A549 calculated from Figure 4 were $2.36 \pm 0.21$ and $1.88 \pm 0.16 \mu \mathrm{g} \cdot \mathrm{mL}^{-1}$, respectively. As a control, the $\mathrm{IC}_{50}$ value of free DOX was $3.69 \pm 0.25 \mu \mathrm{g} \cdot \mathrm{mL}^{-1}$. The antitumor activities increased 1.6-fold $(P<0.01)$ after DOX was encapsulated into LNEs. The enhanced cytotoxicity of DOX-loaded LNE can be attributed to the faster internalization of DOX into cells mediated by nanoemulsion.
Compared with DOX-loaded LNE, PEG-modified DOXloaded LNE also displayed higher cytotoxicity (1.9-fold; $P<0.01$ ), which was probably related to PEG modification promoting the internalization of LNE into cells. ${ }^{23}$

\section{In-vivo biodistribution}

Figure 5 presents the in-vivo fluorescent images of tumorbearing mice after injecting with DiR-labeled LNE through the tail vein. PEGylated LNE can more efficiently accumulate in the tumor area than nonmodified LNE. Regarding DiR/PEG/LNE, most nanoemulsions accumulated in the liver in the first 2 hours. At 8 hours, PEGylated LNE in the liver sharply decreased but significantly increased in

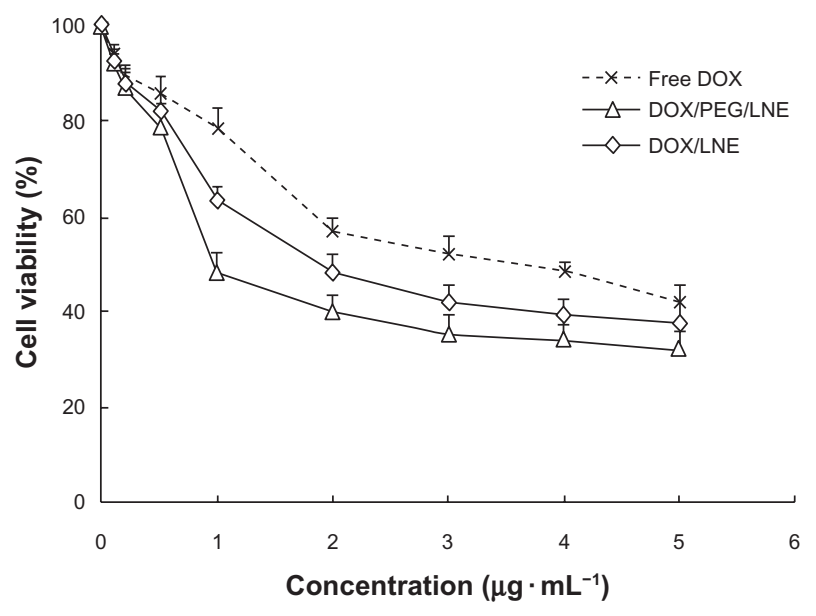

Figure 4 Cell viability after the cells were treated with free DOX, DOX/LNE, and DOX/PEG/LNE with different DOX concentration.

Abbreviations: DOX, doxorubicin; LNE, lipid nanoemulsion; PEG, polyethylene glycol. 

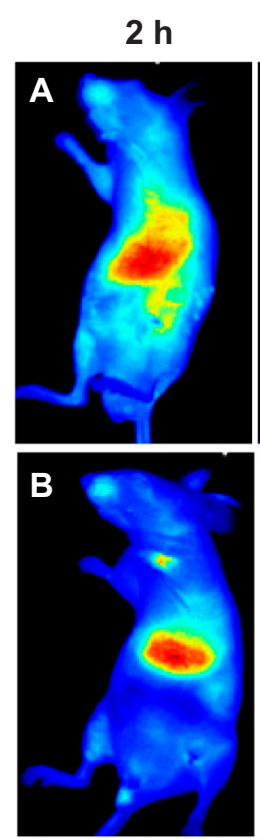

Low
$4 \mathrm{~h}$
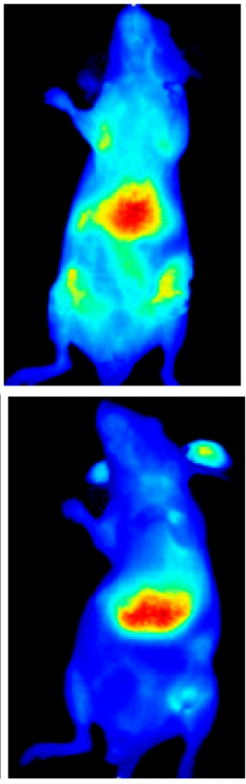

$8 \mathrm{~h}$
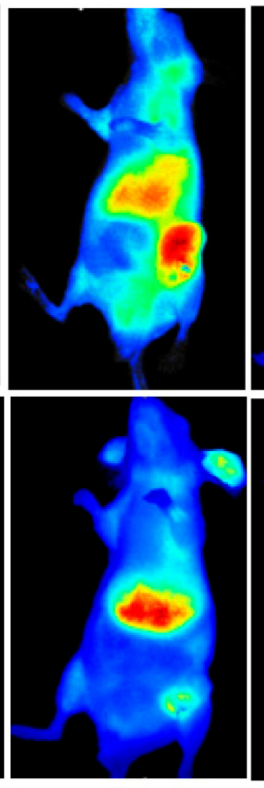

$12 \mathrm{~h}$
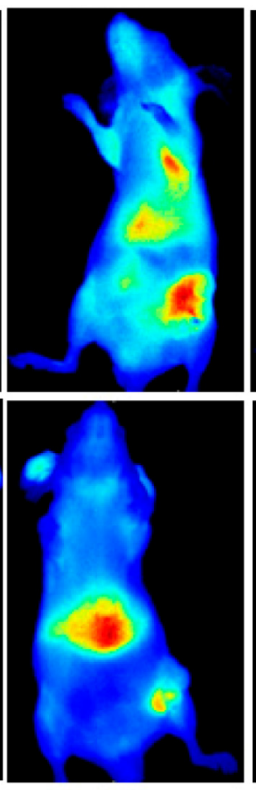

$24 \mathrm{~h}$
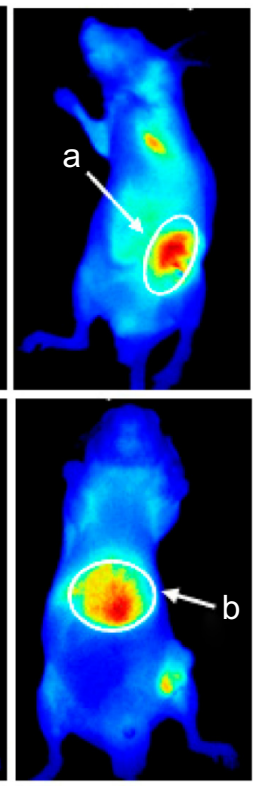

Figure 5 Bio-distribution of DiR-labeled LNE in tumor-bearing mice. Group (A) DiR/PEG/LNE, Group (B) DiR/LNE. Site (a) tumor area, site (b) liver area. Abbreviations: DiR, I, I'-dioctadecyl-3,3,3',3'-tetramethyl indotricarbocyanine iodide; LNE, lipid nanoemulsion; PEG, polyethylene glycol; h, hours.

the tumor region. However, in the DiR/LNE group, most nanoemulsions were distributed in the liver throughout the observation time. The mice were sacrificed 36 hours after injection. Figure 6 shows the in-vivo semi-quantitative
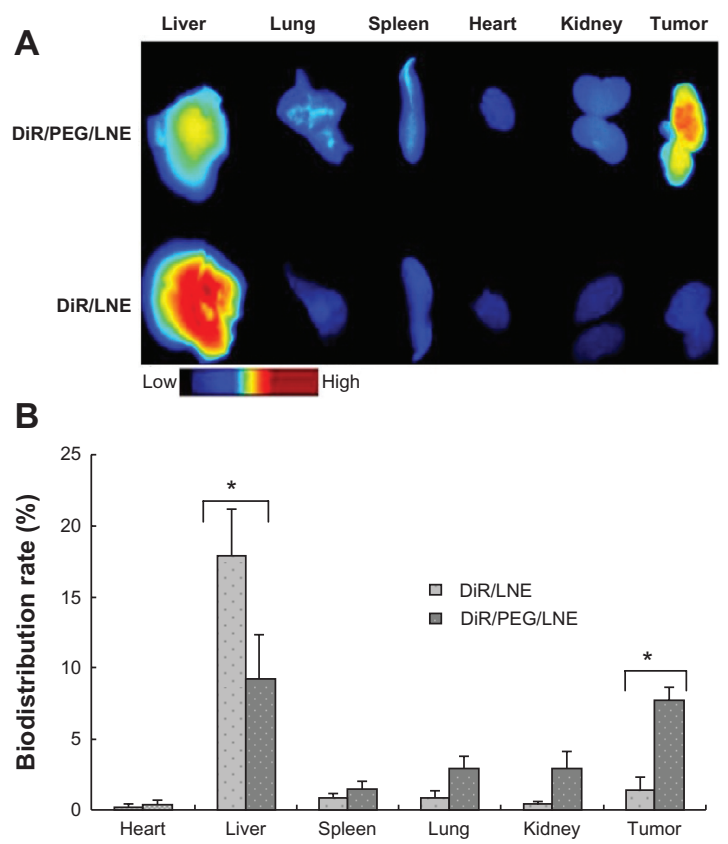

Figure 6 In vivo biodistribution of tumor-bearing mice after DiR-labeled LNE was administrated for 36 hours. Fluorescence images (A) and semi-quantitative biodistribution rate $(\mathbf{B})$.

Abbreviations: DiR, I,I'-dioctadecyl-3,3,3',3'-tetramethyl indotricarbocyanine iodide; LNE, lipid nanoemulsion; PEG, polyethylene glycol.

Note: *Indicates significant difference $(P<0.05)$. biodistribution on tumor-bearing mice after administration of DiR-labeled LNE for 36 hours. The accumulation of LNE in the tumor area increased 6.7 -fold $(P<0.05)$ after PEGylation.

Blood vessels in solid tumor tissue are generally abnormal in form and architecture. They are poorly aligned defective endothelial cells with wide fenestration, ie, lacking a smooth muscle layer or enervated with wider lumen, and impaired functional receptors for angiotensin II. These cells also usually lack effective lymphatic drainage. ${ }^{24,25}$ All these factors lead to the high selectivity and permeability retention of macromolecules and lipid nanoparticles known as high permeability and retention effect of the solid tumor or EPR. ${ }^{26,27}$ However, only particles $<200 \mathrm{~nm}$ in size can passively target the tumor by the EPR effect. More importantly, PEGylation of LNE can exist longer in blood circulation, which can also enhance the EPR effect.

\section{In-vivo antitumor activity}

Adriamycin (a commercial DOX hydrochloride injection), saline, and DOX-loaded LNE were injected through the tail vein into A549 human lung adenocarcinoma-bearing nude mice. Changes in tumor volume and mice weight are shown in Figure 7. Tumor growth can be effectively inhibited by both Adriamycin and drug-loaded LNE. Figure 7B shows a continuous increase in the bodyweight in all groups, which indicated that all doses were within 
A

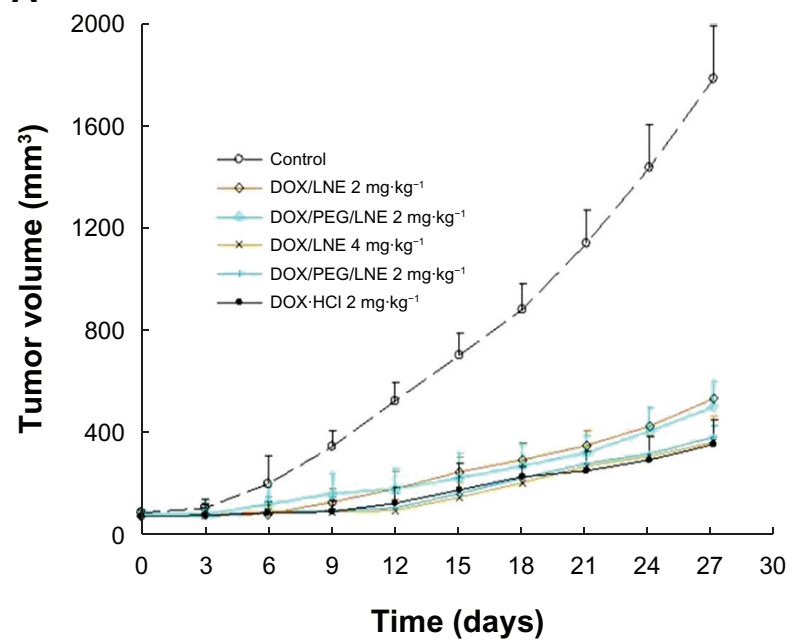

B

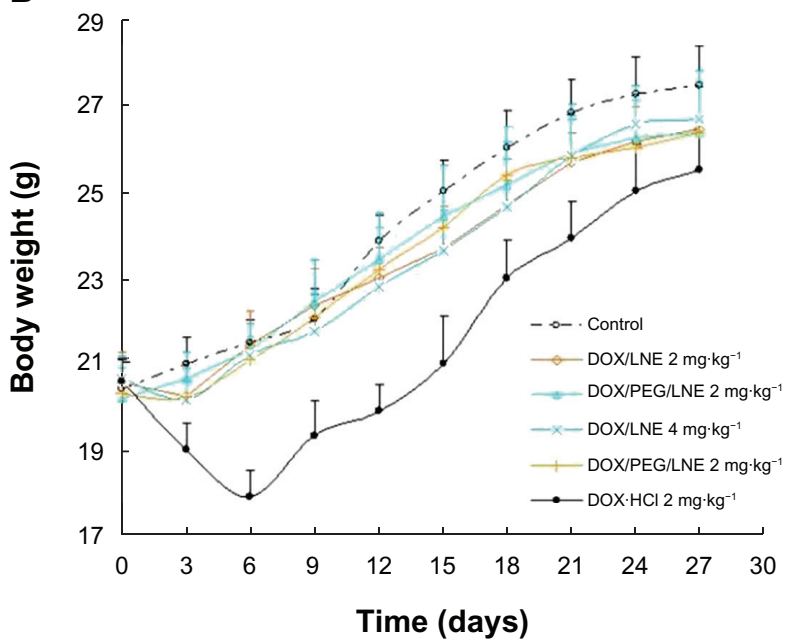

Figure 7 In-vivo antitumor activities of Adriamycin and DOX-loaded LNE in tumor-bearing nude mice: mice tumor volume (A) and mice body weight (B) changed within 27 days $(n=6)$.

Abbreviations: DOX, doxorubicin; LNE, lipid nanoemulsion; PEG, polyethylene glycol.

the safety range. However, the bodyweight of mice sharply decreased in the first 6 days after treatment with Adriamy$\operatorname{cin}\left(2 \mathrm{mg} \cdot \mathrm{kg}^{-1}\right)$.

The tumor inhibition rate was $85.4 \%$ for Adriamycin $\left(2 \mathrm{mg} \cdot \mathrm{kg}^{-1}\right), 73.3 \%$ for DOX/LNE $\left(2 \mathrm{mg} \cdot \mathrm{kg}^{-1}\right), 85.0 \%$ for $\mathrm{DOX} / \mathrm{LNE}, 79.2 \%$ for DOX/PEG/LNE $\left(2 \mathrm{mg} \cdot \mathrm{kg}^{-1}\right)$, and $84.6 \%$ for DOX/PEG/LNE $\left(4 \mathrm{mg} \cdot \mathrm{kg}^{-1}\right)$. The tumor inhibition values of all groups were $>60 \%$, which was considered to be effective for tumor therapy. PEGylation enhanced the
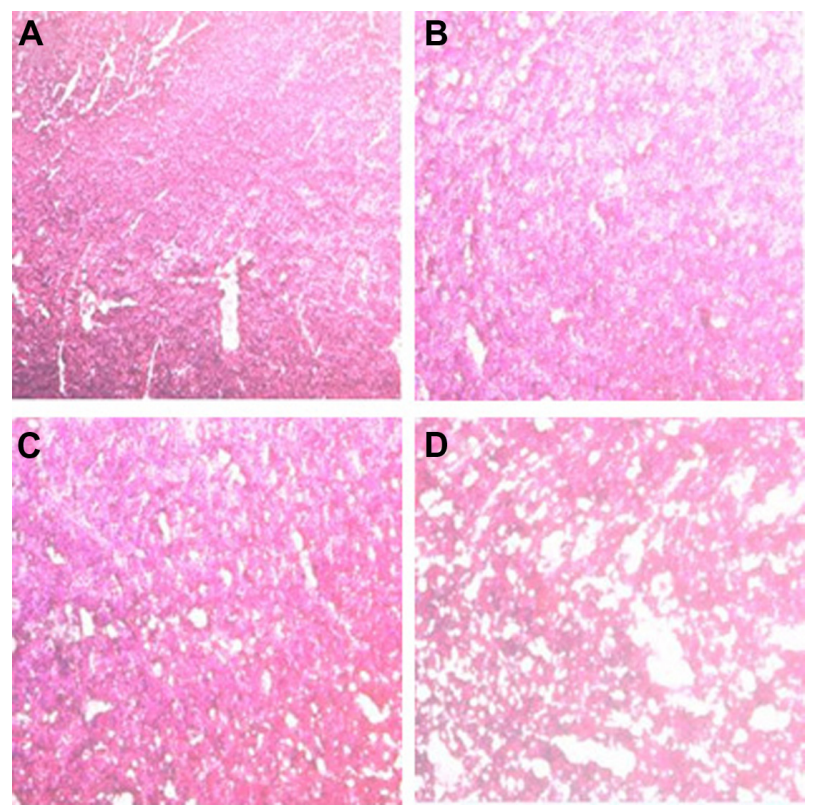

Figure 8 Cardiac tissue obtained from mice which received a single intravenous dose of (A) untreated (as control), (B) normal saline, (C) DOX-loaded LNE, and (D) Adriamycin. Hearts were harvested at day 27 after intravenous injection. Abbreviations: DOX, doxorubicin; LNE, lipid nanoemulsion. tumor inhibition of DOX/LNE at a lower dose. The high dose of Adriamycin $\left(2 \mathrm{mg} \cdot \mathrm{kg}^{-1}\right)$ caused mice death, whereas the LNE-based DDS exerted an equivalent therapeutic effect. This result indicated that DOX-loaded LNE maintained the therapeutic effects of Adriamycin while considerably minimizing its toxicity.

\section{Cardiac toxicity}

Doxorubicin plays an important role in cancer therapy because of its efficacy in treating different types of tumors. However, its clinical use can be limited by the risk of irreversible and possibly life-threatening cardiac injury. Figure 8 shows histopathological changes in heart tissues treated with DOX-loaded LNE had similar results to normal muscle fibers and the saline group (Figure 8A-C). However, as shown in Figure 8D (Adriamycin group), the pathological biopsy of muscle fibers showed severe damage ranging from cavity formation to complete fragmentation. This result indicated that DOX-loaded LNE can reduce the cardiac toxicity of DOX.

\section{Conclusion}

Stable nanosized LNE was successfully prepared by a solvent-diffusion method in an aqueous system. In-vitro drug release from DOX/LNE can be prolonged up to 48 hours. Cellular uptake of LNE was time dependent and can be significantly strengthened by PEGylation. After DOX was encapsulated into LNE, the cytotoxicity increased 1.6-fold and can be further enhanced by PEGylation. LNE can passively accumulate in the tumor tissue and be 
highly enhanced after PEGylation. In-vivo antitumor activity indicated that DOX-loaded LNE can reduce the toxicity level while maintaining the therapeutic effects of commercial DOX hydrochloride. The results suggested that DOX-loaded LNE is a potential candidate for antitumor drug delivery.

\section{Acknowledgments}

This work was sponsored by Zhejiang Provincial Program for the Cultivation of High-level Innovative Health Talents and National Nature Science Foundation of China under contract 81072231 .

\section{Disclosure}

The authors report no conflicts of interest in this work.

\section{References}

1. Deitel M, Friedman KL, Cunnane S, et al. Emulsion stability in a total nutrient admixture for total parenteral nutrition. J Am Coll Nutr. 1992;11:5-10.

2. Gettings SD, Lordo RA, Feder PI, Hintze KL. A comparison of low volume, draize and in vitro eye irritation test data. II. Oil/water emulsions. Food Chem Toxicol. 1998;36:47-59.

3. Mizushima Y. Lipid microspheres (lipid emulsions) as a drug carrierAn overview. Adv Drug Del Rev. 1996;20:113-115.

4. Singh M, Ravin LJ. Parenteral emulsions as drug carrier systems. J Parenter Sci Technol. 1986;40:34-41.

5. Takino T, Konishi K, Takakura Y, Hashida M. Long circulating emulsion carrier systems for highly lipophilic drugs. Biol Pharm Bull. 1994; 17:121-125.

6. Floyd AG. Top ten considerations in the development of parenteral emulsions. Pharm Sci Technol Today. 1999;2:134-143.

7. Palakurthi S, Vyas SP, Diwan PV. Biodisposition of PEG-coated lipid microspheres of indomethacin in arthritic rats. Int $J$ Pharm. 2005;290:55-62.

8. Zhang X, Gan Y, Gan L, Nie S, Pan W. PEGylated nanostructured lipid carriers loaded with 10-hydroxycamptothecin: an efficient carrier with enhanced anti-tumour effects against lung cancer. J Pharm Pharmacol. 2008;60:1077-1087.

9. Moghimi SM, Hunter AC, Murray JC. Long-circulating and targetspecific nanoparticles: theory to practice. Pharmacol Rev. 2001;53: 283-318.

10. Yang T, Choi MK, Cui FD, et al. Preparation and evaluation of paclitaxel-loaded PEGylated immunoliposome. J Control Release. 2007;120:169-177.

11. Lim J, Guo Y, Rostollan CL, et al. The role of the size and number of polyethylene glycol chains in the biodistribution and tumor localization of triazine dendrimers. Mol Pharm. 2008;5:540-547.
12. Liu F, Liu D. Long-circulating emulsions (oil-in-water) as carriers for lipophilic drugs. Pharm Res. 1995;12:1060-1064.

13. Daemen T, Regts J, Meesters M, Ten Kate MT, BakkerWoudenberg IAJM, Scherphof GL. Toxicity of doxorubicin entrapped within long-circulating liposomes. J Control Release. 1997;44:1-9.

14. Maeda H, Wu J, Sawa T, Matsumura Y, Hori K. Tumor vascular permeability and the EPR effect in macromolecular therapeutics: a review. J Control Release. 2000;65:271-284.

15. Webb MS, Saxon D, Wong FM, et al. Comparison of different hydrophobic anchors conjugated to poly(ethylene glycol): effects on the pharmacokinetics of liposomal vincristine. Biochim Biophys Acta. 1998;1372:272-282.

16. Hu FQ, Jiang SP, Du YZ, Yuan H, Ye YQ, Zeng S. Preparation and characterization of stearic acid nanostructured lipid carriers by solvent diffusion method in an aqueous system. Colloids Surf B Biointerfaces. 2005;45:167-173.

17. Hu FQ, Yuan H, Zhang HH, Fang M. Preparation of solid lipid nanoparticles with clobetasol propionate by a novel solvent diffusion method in aqueous system and physicochemical characterization. Int J Pharm. 2002;239:121-128.

18. Texier I, Goutayer M, Da Silva A, et al. Cyanine-loaded lipid nanoparticles for improved in vivo fluorescence imaging. $J$ Biomed l Optics. 2009;14: 054005-054005.

19. Storm G, Belliot SO, Daemen T, Lasic DD. Surface modification of nanoparticles to oppose uptake by the mononuclear phagocyte system. Adv Drug Del Rev. 1995;17:31-48.

20. Bennis S, Chapey C, Robert J, Couvreur P. Enhanced cytotoxicity of doxorubicin encapsulated in polyisohexylcyanoacrylate nanospheres against multidrug-resistant tumour cells in culture. Euro J Cancer. 1994;30:89-93.

21. Gryparis EC, Hatziapostolou M, Papadimitriou E, Avgoustakis K. Anticancer activity of cisplatin-loaded PLGA-mPEG nanoparticles on LNCaP prostate cancer cells. Euro J Pharm Biopharm. 2007;67:1-8.

22. Wan F, You J, Sun Y, et al. Studies on PEG-modified SLNs loading vinorelbine bitartrate (I): preparation and evaluation in vitro. Int $J$ Pharm. 2008;359:104-110.

23. Dadashzadeh S, Vali AM, Rezaie M. The effect of PEG coating on in vitro cytotoxicity and in vivo disposition of topotecan loaded liposomes in rats. Int J Pharm. 2008;353:251-259.

24. Padera TP, Kadambi A, di Tomaso E, et al. Lymphatic metastasis in the absence of functional intratumor lymphatics. Science. 2002;296: 1883-1886.

25. Pepper MS. Lymphangiogenesis and tumor metastasis: myth or reality? Clin Cancer Res. 2001;7:462-468.

26. Hatakeyama $\mathrm{H}$, Akita $\mathrm{H}$, Harashima H. A multifunctional envelope type nano device (MEND) for gene delivery to tumours based on the EPR effect: a strategy for overcoming the PEG dilemma. Adv Drug Del Rev. 2011;63:152-160.

27. Sloat BR, Sandoval MA, Li D, et al. In vitro and in vivo anti-tumor activities of a gemcitabine derivative carried by nanoparticles. Int $J$ Pharm. 2011;409:278-288.
International Journal of Nanomedicine

\section{Publish your work in this journal}

The International Journal of Nanomedicine is an international, peerreviewed journal focusing on the application of nanotechnology in diagnostics, therapeutics, and drug delivery systems throughout the biomedical field. This journal is indexed on PubMed Central, MedLine, CAS, SciSearch ${ }^{\circledR}$, Current Contents ${ }^{\circledR} /$ Clinical Medicine,

\section{Dovepress}

Journal Citation Reports/Science Edition, EMBase, Scopus and the Elsevier Bibliographic databases. The manuscript management system is completely online and includes a very quick and fair peer-review system, which is all easy to use. Visit http://www.dovepress.com/ testimonials.php to read real quotes from published authors. 